\title{
O USO DA CLASSIFICAÇÃO DE COPA DE DAWKINS COMO INDICADOR DO COMPORTAMENTO ECOLÓGICO DE ESPÉCIES ARBÓREAS TROPICAIS
}

\author{
Delman de Almeida Gonçalves ${ }^{1}$, Gustavo Schwartz ${ }^{2}$, Benno Pokorny ${ }^{3}$, Tim van Eldik ${ }^{4}$ \\ ${ }^{1}$ Eng. Agrônomo, M.Sc., Embrapa Amazônia Oriental, Belém, PA, Brasil - delman@ @ cpatu.embrapa.br \\ ${ }^{2}$ Biólogo, M.Sc., Embrapa Amazônia Oriental, Belém, PA, Brasil - gustavo@cpatu.embrapa.br \\ ${ }^{3}$ Eng. Florestal, Ph.D., Universidade de Freiburg, Freiburg, Germany - benno.pokorny@ waldbau.uni-freiburg.de \\ ${ }^{4}$ Eng. Florestal, M.Sc., Ecoflorestal Ltda., Belém, PA, Brasil - tim@ecoflorestal.com \\ Recebido para publicação: 16/11/2006 - Aceito para publicação: 18/05/2009
}

\begin{abstract}
Resumo
O conhecimento do comportamento ecológico de espécies arbóreas de florestas tropicais contribui para a eficiência e aumento da qualidade do manejo florestal. Desse modo, torna-se necessário o desenvolvimento de métodos práticos e eficientes para a classificação ecológica de diferentes espécies de árvores, que possam ser utilizados em levantamentos de curto prazo. Este trabalho analisou o comportamento ecológico quanto ao gradiente de tolerância à sombra, usando o método de Dawkins de classificação da posição e forma da copa, e relacionando esses dados com a fase de desenvolvimento do indivíduo, estimada através da medição do diâmetro à altura do peito (DAP). O estudo foi realizado na área de manejo da empresa Precious Woods Pará (Portel, PA, Brasil), em oito parcelas permanentes de 0,5 ha. Cinco espécies, com diferentes exigências de radiação solar direta, foram analisadas: matamatá-preto (Eschweilera blanchetiana [O. Berg] Miers, Lecythidaceae), maçaranduba (Manilkara huberi (Ducke) Chevalier, Sapotaceae), acapu (Vouacapoua americana Aubl., Fabaceae), andiroba (Carapa guianensis Aubl., Meliaceae) e taxi-preto (Tachigalia myrmecophyla (Ducke) Ducke, Fabaceae). Os resultados encontrados, exceto para maçaranduba, corroboram as informações ecológicas disponíveis na literatura sobre essas espécies, apontando a eficiência do método para essa finalidade.

Palavras-chave: Ecologia florestal; monitoramento florestal; manejo florestal; Amazônia.
\end{abstract}

\begin{abstract}
The Dawkins' crown classification as an indicator of the ecological behavior of tropical tree species. The knowledge about ecological behavior of tree species in tropical forests contributes for the efficiency and quality improvement of forest management. Thus, it makes necessary the development of practical and efficient methods for the ecological classification of different tree species, which can be used in short term surveys. This paper analyzed the ecological behavior as regards to the gradient of shade tolerance, using the Dawkins classification method for crown position and form, and relating these data with the individual developmental phase, which was estimated by the diameter breast height (DBH) measurement. The study was carried out in the management area of Precious Woods Pará company (Portel, PA, Brazil) in eight permanent sample plots of 0.5 ha. Five species having different demand for direct solar radiation were analyzed: matamatá preto (Eschweilera blanchetiana [O. Berg] Miers, Lecythidaceae); maçaranduba (Manilkara huberi (Ducke) Chevalier, Sapotaceae); acapú (Vouacapoua americana Aubl., Fabaceae); andiroba (Carapa guianensis Aubl., Meliaceae); and taxi-preto (Tachigalia myrmecophyla (Ducke) Ducke, Fabaceae). The founded results, excepted for maçaranduba, corroborate the available ecological information about these species in literature, which indicates the method efficiency for this purpose.

Keywords: Forest ecology; forest monitoring; forest management; Amazon.
\end{abstract}

\section{INTRODUÇÃO}

No manejo de florestas tropicais, o conhecimento dos padrões de comportamento ecológico das espécies é de grande importância para a correta tomada de decisões (LUGO; ZIMMERMAN, 2003). 
Segundo Poggiani et al. (1992), cada espécie possui exigências próprias para o seu desenvolvimento. A intensidade de luz que chega ao indivíduo é uma variável determinante ao crescimento e desenvolvimento das espécies florestais, refletindo nos mecanismos de regeneração e crescimento das florestas (JENNINGS et al., 2001). As espécies florestais possuem diferentes comportamentos em relação à intensidade de luz, contribuindo para a definição de suas características sucessionais (DANIEL et al., 1994). A luminosidade também exerce influência sobre todos os estágios de crescimento das plantas, existindo diferentes respostas dos indivíduos para cada fase. As respostas fisiológicas e anatômicas à luz e à escassez de água variam entre as espécies arbóreas e indivíduos de uma mesma espécie, conforme as adaptações a diferentes estágios sucessionais da floresta, dependendo da idade da planta e de sua posição no dossel (RICHARDSON et al., 2000).

Não apenas a quantidade de luz é reduzida nas posições de copa mais baixas e sombreadas, mas a qualidade da luz recebida também é alterada. Segundo King (1996), a alometria de árvores ou, mais especificamente, suas relações entre tamanho e forma, apresenta um relevante efeito estrutural e funcional na floresta. O tamanho, formato e posição de copa de uma árvore relacionam-se com a quantidade de luz interceptada, enquanto que as dimensões do tronco correspondem à sua resistência mecânica frente a forças como o vento e a biomassa da própria copa.

A análise do comportamento de sementes, plântulas e arvoretas de florestas tropicais úmidas, considerando o gradiente de tolerância à sombra, sugere dois grupos ecológicos de espécies: pioneiras e não-pioneiras, estas últimas também chamadas de espécies clímax (SWAINE; WHITMORE, 1988; LUGO; ZIMMERMAN, 2003). A partir de dados de recrutamento e mortalidade de florestas da Malásia, Whitmore (1984) agrupou as espécies de uma floresta madura de acordo com suas estratégias adaptativas e história de vida, sendo (1) espécies pioneiras de vida curta, (2) espécies pioneiras de vida longa sem recrutamento sob um dossel fechado, (3) espécies tolerantes a sombra que não alcançam o dossel superior da floresta, (4) espécies do dossel superior com incremento e (5) espécies do dossel sem incremento. O termo incremento é utilizado, nesse caso, para expressar a continuidade do crescimento da árvore após sua copa ter atingido o dossel. Essa classificação postulou que as árvores emergentes do dossel são demandantes de luz e, eventualmente, se indivíduos das principais espécies de dossel não o atingirem, é porque devem estar em condições de sombreamento. Nesse sentido, formações periódicas de clareiras ou distúrbios de larga escala são fatores importantes na criação de condições para a regeneração da maioria das espécies arbóreas de copa dominante (DENSLOW; HARTSHORN, 1994; WALKER, 2000).

Dawkins (1958) desenvolveu um método de classificação de copa para identificar o gradiente de tolerância à sombra de espécies arbóreas. O método foi inicialmente desenvolvido para florestas tropicais heterogêneas na África (Uganda), com o objetivo de simplificar as atividades de manejo.

Para a região amazônica, o método de classificação de copa de Dawkins, como indicador de tolerância a sombra, pode contribuir para a diagnose do comportamento ecológico de árvores. Com a premissa de que a posição de copa em relação à incidência de luz indica o grau de intensidade de radiação solar que atinge as árvores (OLDEMAN; DIJK, 1991; BROWN et al., 2000), o presente estudo teve como objetivo verificar a validade do método proposto por Dawkins (1958) como indicador prático do comportamento ecológico quanto ao gradiente de tolerância a sombra das espécies florestais, ao longo de suas fases de desenvolvimento.

\title{
MATERIAL E MÉTODOS
}

\author{
Área de estudo \\ O estudo foi realizado na área de manejo florestal da empresa Precious Woods Pará Ltda. \\ $\left(3^{\circ} 23^{\prime} \mathrm{S}\right.$ e $\left.50^{\circ} 17^{\prime} \mathrm{W}\right)$, com altitude média de 19 metros e localizada nos municípios de Portel e Pacajá, \\ estado do Pará, Brasil (Figura 1). A vegetação dominante é de Floresta Ombrófila Densa (VELOSO; \\ GÓES-FILHO, 1982; IBGE, 1992). Na cobertura arbórea ocorrem espécies emergentes, como Dinizia \\ excelsa Ducke, Hymenolobium heterocarpum Ducke e Manilkara huberi (Ducke) Chevalier. O dossel, \\ uniforme, é formado predominantemente por Couratari spp., Parkia spp., Goupia glabra Aubl. e \\ Tachigalia myrmecophylla (Ducke) Ducke. \\ O clima é quente e úmido, do tipo Ami pela classificação de Köppen (tropical chuvoso com \\ pequena estação seca) e do tipo B2rA 'a' pela classificação de Thornthwaite (clima úmido com pequena \\ deficiência hídrica e sem ocorrência de inverno estacional). A temperatura média anual é de $26^{\circ} \mathrm{C}$, sendo
}


a média das temperaturas máximas e mínimas mensais de $32,5^{\circ} \mathrm{C}$ e $22,1{ }^{\circ} \mathrm{C}$, respectivamente. A pluviosidade é de $2.400 \mathrm{~mm}$ por ano, sendo agosto e setembro os meses mais secos, e fevereiro a abril os meses mais chuvosos. O solo predominante é o Latossolo amarelo distrófico de textura média e argilosa. Encontram-se ainda solos concrecionários lateríticos indiscriminados distróficos de textura indiscriminada (EMBRAPA, 1988). O relevo é plano a suave ondulado.

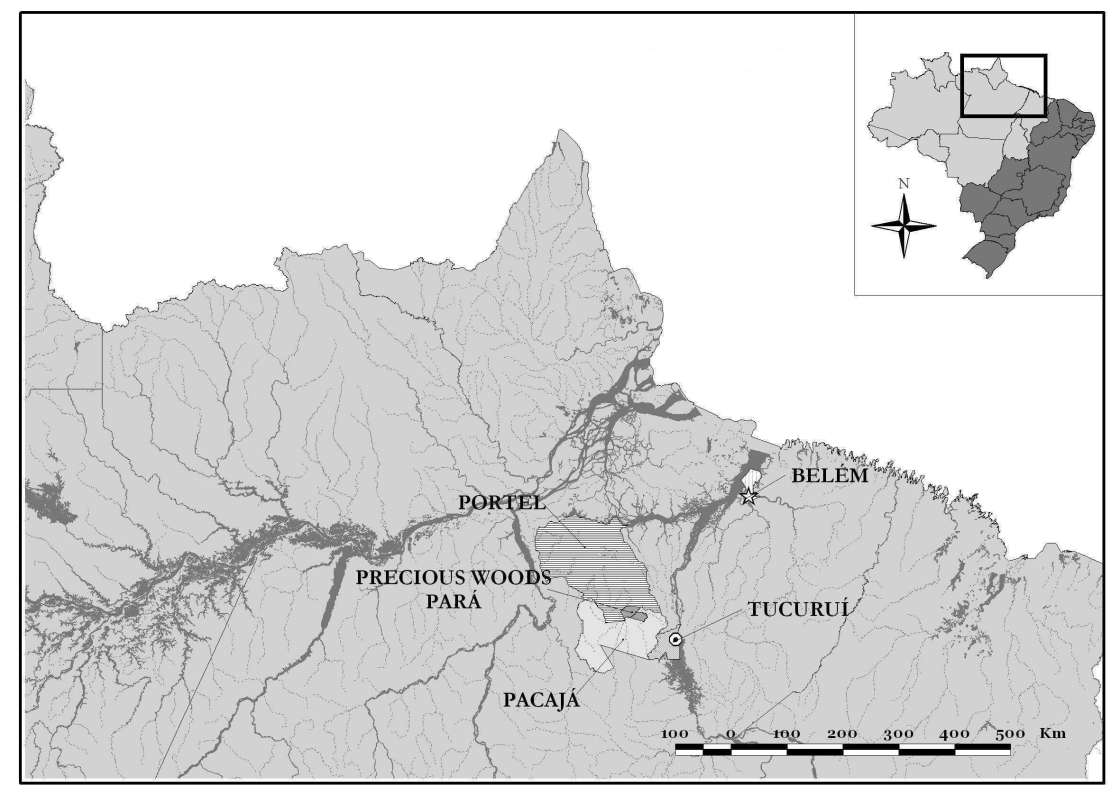

Figura 1. Localização da área de manejo florestal da empresa Precious Woods Pará Ltda. Figure 1. Location of forest management area of Precious Woods Para Ltd. Company.

\section{Amostragem e análise dos dados}

Os dados foram levantados em oito parcelas permanentes de 0,5 ha estabelecidas ao acaso em uma unidade de produção anual (UPA - 2001A), sendo tomados antes da exploração florestal. Todas as observações e avaliações de campo foram feitas pela mesma pessoa. A análise do comportamento ecológico das espécies florestais estudadas foi realizada a partir da observação de duas variáveis de copa, conforme Dawkins (1958): posição e forma.

A posição da copa, conforme Dawkins (1958), é determinada em função da incidência de luz. Para isso, considera-se um cone com ângulo de $90^{\circ}$ a partir da base da copa. O método divide a posição da copa em cinco classes (Figura 2). Na classe 5 (emergente), a superfície da copa está completamente exposta à luz no sentido vertical e está livre de competição lateral; há incidência total de luz no cone. Na classe 4 (iluminação superior completa), a parte superior da copa está completamente exposta à luz, mas ocorre sombreamento lateral de outras copas de altura igual ou maior dentro do cone. Na classe 3 (iluminação superior parcial), a superfície da copa não fica inteiramente exposta à luz no sentido vertical, pois está parcialmente sombreada por outras copas. Na classe 2 (alguma iluminação natural), a superfície da copa está toda sombreada no sentido vertical, mas permanece exposta a alguma luz direta devido a uma clareira ou à extremidade de um dossel superior. Na classe 1 (sem iluminação direta), a superfície da copa está totalmente sombreada, tanto no sentido vertical quanto lateral.

A forma da copa, segundo Dawkins (1958), também é definida em cinco classes (Figura 3). Classe 5 (forma perfeita): nenhuma irregularidade, tanto em perspectiva superior quanto lateral. Classe 4 (boa forma): existe pequena irregularidade no formato da copa. Classe 3 (forma tolerável): as irregularidades são maiores, porém inferiores a $50 \%$ da copa. Classe 2 (forma pobre): as irregularidades são superiores a $50 \%$ da copa. Classe 1 (forma intolerável): as irregularidades são muito grandes, podendo atingir toda a copa. 


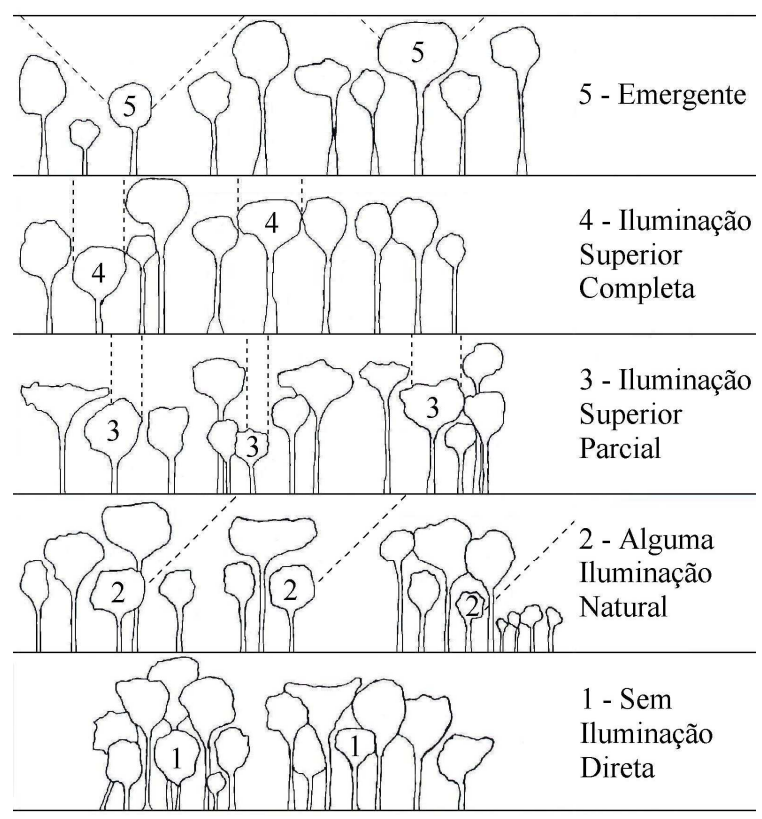

Figura 2. Classificação de posição de copa de Dawkins (modificado de Dawkins, 1958).

Figure 2. Dawkins classification of crown position (modified from Dawkins, 1958).

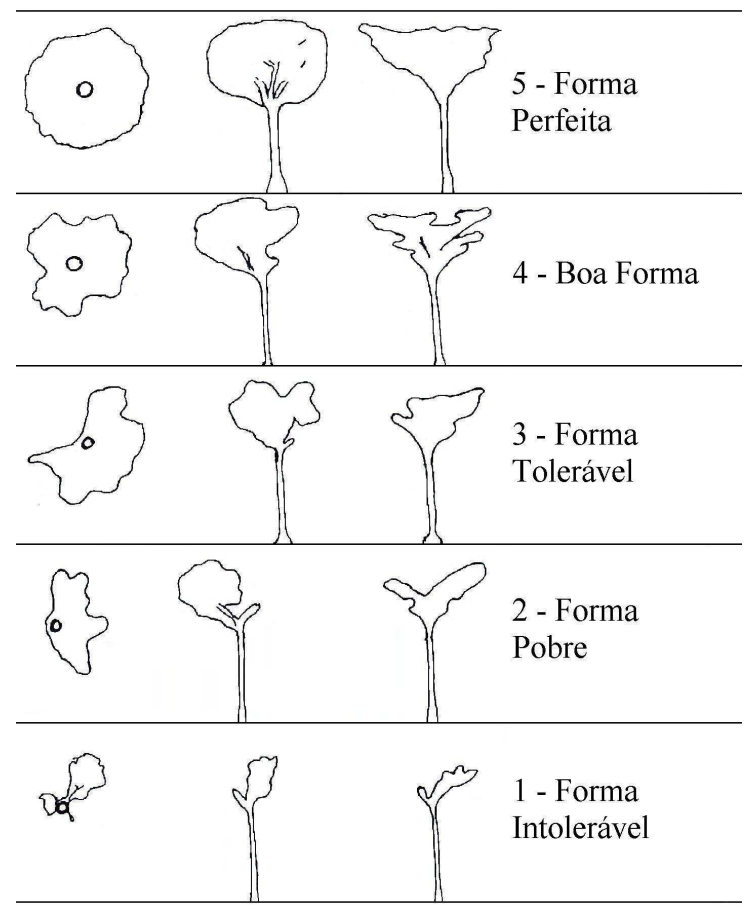

Figura 3. Classificação de forma de copa de Dawkins (modificado de Dawkins, 1958).

Figure 3. Crown shape classification of Dawkins (modified from Dawkins, 1958).

O diâmetro à altura do peito (DAP), ou diâmetro tomado a 1,30 m a partir do solo, foi utilizado como indicador das fases de desenvolvimento das árvores medidas. Os indivíduos encontrados foram divididos em quatro fases de desenvolvimento: fase juvenil $(5 \mathrm{~cm} \leq \mathrm{DAP}<15 \mathrm{~cm})$, fase intermediária 
$(15 \mathrm{~cm} \leq$ DAP $<30 \mathrm{~cm})$, fase pré-adulta $(30 \mathrm{~cm} \leq$ DAP $<50 \mathrm{~cm})$ e fase adulta $($ DAP $\geq 50 \mathrm{~cm})$. O comportamento ecológico quanto ao gradiente de tolerância a sombra das espécies estudadas foi interpretada com base no grau de concentração de indivíduos de cada espécie nas diferentes classes de posição e forma de copa e grupos de diâmetro.

Os dados foram analisados através do teste qui-quadrado $\left(\chi^{2}\right)$, para se verificar a aderência de uma amostra à hipótese nula, neste caso, se as proporções esperadas fossem iguais (AYRES et al., 2005).

\section{Espécies estudadas}

Foram escolhidas cinco espécies para o estudo. Os critérios de seleção foram: 1) representação de diferentes grupos de comportamento ecológico quanto ao gradiente de tolerância a sombra, tomando como base as informações contidas na literatura e a partir da experiência prática de profissionais da área florestal; 2) a existência de valor comercial ou potencialmente comercial de cada espécie; e 3) a ocorrência frequente de indivíduos nas parcelas, para obtenção de uma amostra representativa. Este critério foi determinante no trabalho, devido às baixas densidades encontradas para as espécies inventariadas, o que traduz o padrão das florestas tropicais densas (HUBBELL; FOSTER, 1986; HUSTON, 1995). As espécies escolhidas, seguindo um gradiente de maior para menor tolerância a sombra, foram: matamatá-preto (Eschweilera blanchetiana (O. Berg) Miers, Lecythidaceae), maçaranduba (Manilkara huberi (Ducke) Chevalier, Sapotaceae), acapu (Vouacapoua americana Aubl., Fabaceae), andiroba (Carapa guianensis Aubl., Meliaceae) e taxi-preto (Tachigalia myrmecophyla (Ducke) Ducke, Fabaceae).

\section{RESULTADOS E DISCUSSÃO}

Foram amostrados 126 indivíduos, sendo as espécies mais abundantes o matamatá-preto e o acapu, seguidos por andiroba, taxi-preto e maçaranduba. As fases de desenvolvimento mais abundantes foram a pré-adulta e a intermediária, seguidas pelas fases juvenil e adulta (Tabela 1).

Tabela 1. Número de indivíduos em cada fase de desenvolvimento para as cinco espécies estudadas na área de manejo florestal da Precious Woods Pará.

Table 1. Number of individuals in each developmental phase for five studied species in the forest management area of Precious Woods Para.

\begin{tabular}{lccccc}
\hline \multirow{2}{*}{ Espécie } & \multicolumn{4}{c}{ Fase de desenvolvimento } & \multirow{2}{*}{ Total } \\
\cline { 2 - 5 } & Juvenil & Intermediária & Pré-adulta & Adulta & \\
\hline Matamatá-preto & 14 & 16 & 20 & 0 & 50 \\
Maçaranduba & 1 & 2 & 4 & 2 & 9 \\
Acapu & 7 & 18 & 15 & 6 & 46 \\
Andiroba & 0 & 1 & 7 & 4 & 12 \\
Taxi-preto & 2 & 3 & 1 & 3 & 9 \\
\hline
\end{tabular}

Os indivíduos de matamatá-preto concentraram-se nas posições 1 e 2 e com formas de copa nas classes 3 e 4 (Tabela 2). Isso indica um alto grau de tolerância a sombra em todos os estágios de desenvolvimento, especialmente por apresentar a maior parte dos indivíduos com boa forma de copa. Os indivíduos dessa espécie chegaram, no máximo, à posição de dossel intermediário no povoamento florestal, recebendo, em sua maioria, apenas luz lateral nas copas, o que indica adaptação da espécie ao sombreamento contínuo. Isso corrobora os trabalhos de Pinheiro et al. (2007), Carvalho (2000) e Pereira; Pedroso (1972), ao classificarem o matamatá-preto como tolerante a sombra, de crescimento lento e com boa regeneração natural, atingindo 10 a $15 \mathrm{~m}$ de altura, com copa pequena e estreita.

O acapu foi a espécie mais abundante nas classes 2 e 3 quanto à posição de copa e na classe 3 para a forma (Tabela 2). Esses resultados intermediários a baixos, tanto para a posição quanto para a forma de copa, indicam a existência de adaptações para a tolerância a sombra: é uma espécie com tolerância à sombra no estágio juvenil e que, gradualmente, exige mais luz à medida que cresce. No entanto, foi observado neste estudo que indivíduos que chegam ao dossel mais elevado ocupam sua porção média, com as copas parcialmente encobertas, indicando permanência do comportamento de

FLORESTA, Curitiba, PR, v. 40, n. 1, p. 175-182, jan./mar. 2010. 
tolerância a sombra na fase adulta. O acapu pode alcançar de 15 a 25 metros de altura (SUDAM, 1979) e ocorre principalmente em matas primárias de terra firme, comportando-se como espécie clímax (SOUZA et al., 1998). Os resultados obtidos estão de acordo com Maués et al. (1999), que indicaram o acapu como espécie tolerante a sombra quando jovem, instalando-se como secundária tardia em processos de sucessão e atingindo o dossel na idade adulta.

Tabela 2. Posição e forma predominante de copa das cinco espécies estudadas na área de manejo florestal da Precious Woods Pará.

Table 2. Predominant crown position and shape for five studied species in the forest management area of Precious Woods Pará.

\begin{tabular}{lcc}
\hline Espécie & Posição de copa & Forma de copa \\
\hline Matamatá-preto & 1 e $2 * *$ & 3 e $4 * *$ \\
Maçaranduba & n.s. & n.s. \\
Acapu & 2 e $3 * *$ & $3 * *$ \\
Andiroba & 2 e $3 * *$ & $2 *$ \\
Taxi-preto & $4 *$ & 3 e $4 * *$ \\
\hline$*$ Valores de $\chi^{2}$ significativos a 5\%; ** a $1 \%$ e não significativos (n.s.) a 5\% de probabilidade \\
de erro do tipo I.
\end{tabular}

A andiroba apresenta baixa tolerância a sombra no estágio juvenil, o que foi evidenciado pela ausência de indivíduos na classe de menor DAP (Tabela 1). Algumas espécies que ocorrem na floresta adulta necessitam muita luz em seu estágio inicial de desenvolvimento, e a deficiência de luminosidade causada pela cobertura de uma floresta densa torna-se prejudicial à sua regeneração natural (CARVALHO, 1982). Os indivíduos no estágio adulto mostraram maior tolerância a sombra, ocorrendo nas classes de posição de copa 2 e 3 . No entanto a maioria das árvores amostradas apresentaram forma de copa pobre (classe 2), indicando intolerância a sombra (Tabela 2). A má qualidade de forma de copa pode estar indicando deformidades devido à falta de luz direta em indivíduos de espécie não adaptada a ambientes sombreados.

A literatura sobre andiroba é controversa. Pereira; Pedroso (1972) citam que a andiroba pertence ao estrato subdominante da floresta, atingindo de 15 a $25 \mathrm{~m}$ e, eventualmente, chegando a $30 \mathrm{~m}$, com copa média e ramosa. Finegan (1992) classificou-a como espécie esciófita parcial, ou seja, comporta-se como tolerante a sombra nas etapas iniciais de desenvolvimento. No entanto as mudas crescem rápido sob iluminação direta, acelerando o processo na presença de aberturas no dossel. Sendo assim, toleram a sombra mas não dependem dela para o seu desenvolvimento. Em situações experimentais, a andiroba apresentou comportamento típico de espécies secundárias tardias (SILVA et al., 1996). Parrota; Francis (1995) citam que a andiroba é uma árvore de porte médio, geralmente atingindo posição de dossel médio ou superior. Carvalho (2000) a classifica como tolerante a sombra.

Pereira; Pedroso (1972) citam que o taxi-preto pertence ao estrato subdominante da floresta primária, atingindo geralmente 15 a 25 metros, com copa grande e larga. É descrito como uma árvore de porte médio que, geralmente, alcança o dossel superior em florestas secundárias (PARROTA; FRANCIS, 1995), evidenciando baixa tolerância a sombra. A espécie pode apresentar alguma tolerância a sombra no estágio juvenil (observação pessoal), porém os resultados mostram diferença significativa em favor de indivíduos sujeitos à plena luz vertical (classe 4). A baixa tolerância a sombra fica ainda mais evidenciada devido aos indivíduos apresentarem boa forma de copa, nas classes 3 e 4 (Tabela 2).

A maçaranduba não apresentou nenhuma diferença significativa em favor de alguma forma ou posição de copa em especial (Tabela 2), apesar de os indivíduos na fase adulta estarem no dossel superior da floresta. A maçaranduba é uma árvore do estrato dominante da floresta (PEREIRA; PEDROSO, 1972), com alturas entre 30 e 40 metros (SUDAM, 1979; EMBRAPA, 2004). Em florestas primárias, ela alcança posição superior no dossel ou mesmo emergente (PARROTA; FRANCIS, 1995). Embora Pinheiro et al. (2007) definam a maçaranduba como tolerante a sombra, os resultados encontrados neste trabalho não permitem definir seu padrão de comportamento ecológico através do método de Dawkins (Tabela 2).

Apesar das dificuldades para a classificação das espécies arbóreas em grupos ecológicos por meio de indicadores simples (GUZMÁN, 1997), a metodologia de Dawkins mostra-se como ferramenta prática e eficiente para a avaliação do comportamento ecológico de espécies florestais nos estágios juvenis a adultos, auxiliando em decisões sobre manejo florestal. Com exceção da maçaranduba, de 
comportamento indiferente, para as demais espécies estudadas os resultados encontrados foram corroborados pela literatura. No entanto, essa metodologia ainda precisa ser testada em mais espécies de ecologia conhecida antes de ser utilizada no manejo florestal de escala comercial.

\section{REFERÊNCIAS}

AYRES, M.; AYRES Jr., M.; AYRES, D. L.; SANTOS, A. S. BioEstat 4.0: aplicações estatísticas nas áreas das ciências biológicas e médicas. Belém: Mamirauá/ MCT/ Imprensa Oficial do Estado do Pará. 2005. $324 \mathrm{p}$.

BROWN, N.; JENNINGS, S.; WHEELER, P.; NABE-NIELSEN, J. An improved method for the rapid assessment of forest understorey light environments. Journal of Applied Ecology, London, v. 37, n. 6. p. 1044-1053. 2000.

CARVALHO, J. O. P. Análise estrutural da regeneração natural em floresta tropical densa na região de Tapajós no Estado do Pará. 128 f. Dissertação (Mestrado em ciências Florestais) Universidade Federal do Paraná, Curitiba, 1982.

CARVALHO, J. O. P. Classificação em grupos ecológicos das espécies mais importantes em uma área de terra firme da Floresta Nacional do Tapajós. Belém: Embrapa Amazônia Oriental, 2000. 4 p. (Comunicado Técnico, n. 41).

DANIEL, O.; OHASHI, S. T.; SANTOS, R. A. Produção de mudas de Goupia glabra (Cupiúba): efeito de níveis de sombreamento e tamanho de embalagens. Revista Árvore, Viçosa,MG, v. 18, n. 1, p. 1-13, 1994.

DAWKINS, H. C. The management of natural tropical high forest with special reference to Uganda. Oxford: University of Oxford, Imperial Forestry Institute, 1958. 155 p.

DENSLOW, J. S.; HARTSHORN, G. S. Tree-fall gap environments and forest dynamic processes. In: MCDADE, L. A.; BAWA, K. S.; HESPENHEIDE, H. A.; HARTSHORN, G. S. La Selva: ecology and natural history of a neotropical rain forest. Chicago: University of Chicago Press. 1994. p. 120-127.

EMBRAPA. Serviço Nacional de Levantamento e Conservação de Solo. Critérios para distinção de classes de solos e de fases de unidades de mapeamento: normas em uso pelo SNLCS. Rio de Janeiro, 1988. 67 p. (Embrapa-SNLCS. Documentos, 11).

EMBRAPA AMAZÔNIA ORIENTAL. Maçaranduba, Manilkara huberi. Belém, 2004. 6 p. (Espécies arbóreas da Amazônia, n.4).

FINEGAN, B. El potencial de manejo de los bosques húmedos secundarios neotropicales de las tierras bajas. Turrialba: CATIE. 1992. 29 p.

GUZMÁN, R. A. G. Consideraciones teoricas y metodologias practicas para la asignacion de gremios ecologicos para las especies forestales de bosques humedos tropicales. Disponível em: <http://bolfor.chemonics.net/bolfor/DOCUMENT/DT58E.PDF>. Acesso em: 15/10/2006.

HUBBELL, S. P.; FOSTER, R. B. Commonness and rarity in a neotropical forest: implications for tropical tree conservation. In: SOULÉ M.E. (Ed.). Conservation Biology: the science of scarcity and diversity. Massachusetts: Sinauer. 1986. p. 205-231.

HUSTON, M. A. Biological diversity: the coexistence of species on changing landscapes. New York: Cambridge University Press. 1995. 681 p.

IBGE - INSTITUTO BRASILEIRO DE GEOGRAFIA E ESTATÍSTICA. Atlas nacional do Brasil. Brasília, DF. Disponível em: <http://www2.ibge.gov.br/pub/Cartas_e_Mapas/Mapas_Tematicos>. Acesso em: 15/10/2006.

JENNINGS, S. B.; LOPES, J. C. A.; WHITMORE, T. C.; BROWN, N. D. Dinâmica da regeneração natural de algumas espécies florestais na Floresta Nacional do Tapajós, Estado do Pará, Brasil. In: SILVA, J. N. M.; CARVAlHO, J. O. P.; YARED, J. A. G. (Eds.). A silvicultura na Amazônia Oriental. Belém: Dfid/ Embrapa. 2001. p. 253-274. 
KING, D. A. Allometry and life history of tropical trees. Journal of Tropical Ecology, Cambridge, v. 12, p. 25-44, 1996.

LUGO, A. E.; ZIMMERMAN, J. K. Ecological life histories of tropical trees with emphasis on disturbance effects. In: VOSSO, J. (Ed. ). Tropical tree seed manual. Washington, DC: USDA Forest Service. 2003. p. $191-213$.

MAUÉS, M. M.; SANTOS, L. F. C.; MACQUEEN, D.; MARTINS-DA-SILVA, R. C. V. Biologia da polinização do acapu (Vouacapoua americana Aubl. Leguminosae), uma essência florestal amazônica. In: SIMPÓSIO SILVICULTURA NA AMAZÔNIA ORIENTAL - Contribuições do Projeto Embrapa/DFID, 1999, Belém. Resumos expandidos. Belém: Embrapa Amazônia Oriental/DFID. 1999. p.15-19.

OLDEMAN, R. A.; DIJK, J. Diagnosis of the temperament of tropical rain forest trees. In: GÓMEZPOMPA, A.; WHITMORE, T. C.; HADLEY, M. (Eds.): Rain forest regeneration and management. Paris: UNESCO e Parthenon, 1991. v. 6, p. 21-65.

PARROTA, J. A.; FRANCIS, J. K. Trees of the Tapajós: a photographic field guide. Rio Piedras: International Institute of Tropical Forestry. 1995. 371 p.

PEREIRA, A. P.; PEDROSO, L. M. Experimentos de Silvicultura Tropical: I. Informações sobre épocas de floração, frutificação e desfolha das principais essências00000 florestais que ocorrem na Estação Experimental de Curuá-Una, Estado do Pará. Belém: SUDAM. 1972. 69 p.

PINHEIRO, K. A. O.; CARVALHO, J. O. P.; QUANZ, B.; FRANCEZ, L. M. B.; SCHWARTZ, G. Fitossociologia de uma área de preservação permanente no leste da Amazônia: indicação de espécies para recuperação de áreas alteradas. Revista Floresta, Curitiba, v. 37, n. 2, p. 175-187, 2007.

POGGIANI, F.; BRUNI, S.; BARBOSA, E. S. Q. Efeito do sombreamento sobre o crescimento das mudas de três espécies florestais. Revista do Instituto Florestal de São Paulo, Piracicaba, v. 4, n. 2, p. 564-569, 1992.

RICHARDSON, A. D.; BERLYN, G. P.; ASHTON, P. M. S.; THADANI, R.; CAMERON, I. R. Foliar plasticity of hybrid spruce in relation to crown position and stand age. Canadian Journal of Botany, Ottawa, v. 78, M. 3, p. 305-317, 2000.

SILVA, R. F. A. G.; PIÑA-RODRIGUES, F. C. M.; COSTA, L. G. S. Comportamento de crescimento de espécies arbóreas em plantios na Amazônia. In: CONGRESSO INTERNACIONAL DE COMPENSADO E MADEIRA TROPICAL, 2. 1996, Rio de Janeiro. Anais... Rio de Janeiro: Senai/DN, 1996. p. 297.

SOUZA, L. A. G.; DANTAS, A. R.; MATOS, R. B.; SILVA, M. F. F. Período de frutificação e variabilidade das sementes de acapú (Vouacapoua americana Aubl. - Leg. - Caesalpinioidea) coletadas na região do médio rio Tocantins, Pará. In: REUNIÃO DE BOTÂNICOS DA AMAZÔNIA, 2., 1998, Belém. Programas e Resumos. Belém: Museu Paraense Emílio Goeldi. 1998. p. 38-39.

SUDAM - SUPERINTENDÊNICA DO DESENVOLVIMENTO DA AMAZÔNIA. Centro de Tecnologia Madeireira. Pesquisas e informações sobre espécies florestais da Amazônia. Belém, 1979. $110 \mathrm{p}$.

SWAINE, M. D.; WHITMORE, T. C. On the definition of ecological species groups in tropical rain forests. Vegetatio, Oslo, v. 75, p. 81-86, 1988.

VELOSO, H. P.; GÓES-FILHO, L. Fitogeografia brasileira: classificação fisionômico-ecológica de vegetação tropical. Salvador: Projeto Radambrasil. 1982. 85 p. (Série Vegetação Salvador, n.1).

WALKER, L. R. Seedling and sapling dynamics of treefall pits in Puerto Rico. Biotropica, Malden, v. 32, n. 2, p. 262-275. 2000.

WHITMORE, T. C. Tropical rain forests of the Far East. Oxford: U.K.:Clarendon Press, 1984. 352 p. 